\title{
On the capabilities of the inaction method for extracting the periodic components from GPS clock data
}

\author{
Guocheng Wang ${ }^{1} \cdot$ Lintao Liu $^{1} \cdot$ Aigong Xu${ }^{2} \cdot$ Feng $\mathrm{Pan}^{3} \cdot$ Zhiwu Cai $^{3} \cdot$ Shenghong Xiao ${ }^{3} \cdot \mathrm{Yi} \mathrm{Tu}^{4} \cdot$ Zhonghua Li $^{1}$
}

Received: 4 September 2017 / Accepted: 1 July 2018 / Published online: 6 July 2018

(c) The Author(s) 2018

\begin{abstract}
Almost all GPS signal clocks show a periodic fluctuation. Harmonics in GPS satellites are a well-known feature, such as 3-, 4-, 6- and 12-h terms, accurate extraction of these harmonics are helpful to improve the clock modeling. The inaction method (IM) originating from the concept of normal time-frequency transform (NTFT) can precisely extract the periodic signals which significantly present the NTFT spectrum. This method is essentially line-pass filtering, so it can avoid being polluted by noises to the maximum extent and then have the ability to extract the time-varying periodic signals with robustness. The simulation test demonstrates that IM is the best method for extracting the harmonics and time-varying harmonics from a time signal, compared to singular spectrum analysis and zero-phase digital filter. We focus on GPS satellite clock data, after removing 6- and 12-h terms extracted by IM, the oscillation phenomenon in Hadamard deviation virtually disappears; this demonstrates that these periodic signals have been extracted effectively by the IM.
\end{abstract}

Keyword GPS satellite clocks · Normal time-frequency analysis $\cdot$ Inaction method $\cdot$ Harmonic analysis $\cdot$ Hadamard deviation

\section{Introduction}

The onboard atomic frequency standard (AFS) is a fundamental element of a GNSS satellite, it plays an important role in the determination of the user position and characterizing the timing performance of the GNSS satellites, thus the satellite clock stability directly affects the range measurement and limits the navigation accuracy (Senior et al. 2008; Cernigliaro et al. 2013a, b; Hauschild et al. 2013). Thus, it is meaningful to establish the GNSS clock models with high precision for the GNSS positioning and timing capabilities.

In GNSS, each satellite of the constellation carries more than one atomic clock but one controls all the timing

Lintao Liu

1lt@whigg.ac.cn

1 State Key Laboratory of Geodesy and Earth's Dynamics, Institute of Geodesy and Geophysics, Chinese Academy of Sciences, CAS, Wuhan 430077, China

2 Liaoning Technical University, PRC, Fuxin 123000, China

3 Beijing Satellite Navigation Center, Beijing 100094, China

4 Key Laboratory of Geological Hazards on Three Gorges Reservoir Area, Ministry of Education, China Three Gorges University, Yichang 443002, China operations, such as signal generation and broadcasting, GNSS satellites employ different clock technologies: the GPS uses Cesium clocks and Rubidium Atomic clocks, GLONASS uses Cesium clocks, whereas Galileo and the BeiDou use RAFS and passive hydrogen masers (PHM) (Cernigliaro et al. 2013a, b; Hauschild et al. 2013). The current GPS constellations are running four families of clocks as shown in Table 1: the Block IIR and Block IIR-M satellites carry rubidium clocks, exclusively (Hauschild et al. 2013). The newest generation of Block IIF satellites uses improved Rubidium clocks, which has demonstrated superior stability in ground tests, and improved Cesium clocks (Vannicola et al. 2010; Weiss et al. 2010; Montenbruck et al. 2012). Finally, the next generation of Block IIIA satellites will be launched in near future (Marquis and Reigh 2015).

The International Global Navigation Satellite Systems Service (IGS) operates a global network of Global Navigation Satellite System (GNSS) ground stations, data centers, and data analysis centers to provide data and derived data products to satisfy the objectives of a wide range of applications and experimentation, such as earth science research, multi-disciplinary positioning, navigation, and timing (PNT) applications; and education (IGS 2013). The IGS published the Broadcast, Ultra-Rapid, Rapid and Final 
Table 1 Satellite information of the GPS system (as of July 2, 2016)

\begin{tabular}{lll}
\hline Block-type & Clock & PRN \\
\hline IIR & RB & $2,11,13,14,16,18,19,20,21,22,23,28$ \\
IIR-M & RB & $5,712,15,17,29,31$ \\
IIF & RB & $1,3,6,9,10,25,26,27,30,32$ \\
IIF & CS & 8,24 \\
\hline
\end{tabular}

Contents extracted from the website 'http://www.navcen.uscg. gov/?Do=constellationStatus'

products. Among them, the IGS Final products have the highest quality and internal consistency of all IGS products, which provide an opportunity for detecting the GPS clock characteristic with higher accuracy and finer temporal resolution (Senior et al. 2008).

Periodic fluctuations are apparently present in almost all GPS signal clocks (Gonzalez et al. 2010; Gonzalez Martinez 2013; Galleani and Tavella 2016). Some studies attribute these periodic fluctuations to the residuals of imperfect orbit estimation or the influence of periodic factors such as temperature and environment (Heo et al. 2010; Sesia et al. 2011; Zhou et al. 2011; Cernigliaro et al. 2013a, b). The periodic variations are very important because they can be used to improve the clock modeling, including interpolation of tabulated IGS products for higher-rate GPS positioning and predictions in real-time applications. This is especially true for high-accuracy uses but could also benefit the standard GPS operational products (Senior et al. 2008). Some tools are used to detect these periodic fluctuations, such as the dynamic allan variance, power spectrum and time-frequency spectrum (Galleani 2008; Sesia et al. 2011; Amin et al. 2017).

Conventionally, the periodic and time-varying periodic signals also called the harmonics and time-varying harmonics, are modeled by least-squares fitting harmonic terms with constant amplitudes and frequency. However, the time-varying harmonics have time-variable amplitudes and phases (Chen et al. 2013). Band-pass filtering, such as Fourier transform (Popinski and Kosek 1995), wavelet transform (Liu et al. 2007; Chao et al. 2014) and zero-phase digital filter (ZPDF) are often used to extract the periodic or time-varying periodic with time-variable amplitude and frequency (Höpfner 2004). But the time-varying periodic obtained by these methods will be corrupted easily by noise components, especially in the case when a large amounts of noise exist in the mixed signals because these methods choose a frequency band range in which may exist a lot of noises. In addition, other spatio-temporal analysis methods are also used to extracting the time-varying periodic signals, such as the singular spectrum analysis (SSA) (Chen et al. 2013), and empirical mode decomposition (EMD) (Smylie et al. 2015). However, when implementing the SSA, a key problem is choosing an appropriate lag-window size that determines the spectral resolution of the algorithm (Chen et al. 2013); EMD has the problem of mode mixing (Wu and Huang 2009). To overcome these problems, we present the inaction method (IM) and its principle. The inaction method, which is essentially line-pass filtering, can avoid polluting by noises to the maximum extent when extracting the timevarying periodic signals.

In GPS satellites, harmonics are a well-known feature since the early estimations of GPS clock (Swift and Hermann 1988; Senior et al. 2008; Gonzalez Martinez 2013). Four harmonic frequencies have been detected in all GPS clock types, $n \times(2.0029 \pm 0.0005)$ cycles per day (24-h coordinated universal time or UTC), for $n=1,2,3$, and 4 (Senior et al. 2008).

The GPS satellite clock data used in this study comes from the IGS Final $300 \mathrm{~s}$ satellite clocks (ftp://cddis.gsfc. nasa.gov/pub/gps/products/), active during 8 weeks from May 8 to July 2, 2016. The satellite information can be seen in Table 1 for this time range. PRN04 is not included in this table because it contains lots of missing data or abnormal values during this time range.

In the next section, we introduce the concept of the normal time-frequency transform and present the inaction principle which is the theoretical basis of the IM. Subsequent sections compare the extraction capacity of IM, SSA, and ZPDF from simulation time series data, and then we focus particular attention on the amplitude variations of 6- and 12-h terms extracted by the IM from the GPS satellite clock data over time. Finally, we compare the Hadamard deviation of the satellite clocks before and after exacting the 6- and 12-h terms.

\section{Normal time-frequency transform}

Time-frequency analysis can identify the instantaneous frequency, phase and amplitude of the undulation, which has been used in detecting periodic fluctuations. For unbiased measurement of the instantaneous frequency, phase and amplitude of a time series, the normal time-frequency transform (NTFT) has been proposed by Liu and Hsu (2009, 2011). Refer to these studies for detailed proof and properties of the NTFT explained below.

Definition For time function $f(t)$, where $f: R \rightarrow C$, its timefrequency transform

$\Psi f(\tau, \varpi)=\int_{R} f(t) \bar{\psi}(t-\tau, \varpi) \mathrm{d} t, \tau, \varpi \in R$

is called a NTFT, if the Fourier Transform of the transform kernel $\psi(t, \varpi)$ 
$\hat{\psi}(\omega, \varpi)=\int_{R} \psi(t, \varpi) \exp (-i \omega t) d t$

satisfies

(i) $\hat{\psi}(\omega, \varpi)=1$, if $\omega=\varpi$

(ii) $|\hat{\psi}(\omega, \varpi)|<1$, if $\omega \neq \varpi$

where $\tau$ is the time index and $\varpi$ the frequency index, the line '-' denotes the conjugate operator, $C$ is the complex field and R the real field; and "Il" is the modulus operator.

Satisfying (1), a typical NTFT kernel can be constructed as:

$\psi(t, \varpi)=|\mu(\varpi)| w(\mu(\varpi) t) \exp (i \varpi t), w(t) \in \Omega(R),(\mu(\varpi) \in R) \neq 0$

where $\Omega(R)$ is the function space of all the normal windows $w(t)$. The rescaling index $\mu(\varpi)$ can be almost anything other than zero, for instance, $\varpi, \varpi^{2 / 3}, \varpi^{5}$ and so on. Particularly, if letting $\mu(\varpi)=1$, Eq. (2) yields a phase-updated Gabor transform (GT) and letting $\mu(\varpi)=\varpi$ yields a normal wavelet transform (NWT).

$\Psi f(\tau, \varpi)=|\varpi| \int_{-\infty}^{+\infty} f(t) w(\varpi(t-\tau)) \exp (-i \varpi(t-\tau)) d t \tau, \varpi \in R$

In (5) and (6), the local time function $w(t) \in L^{1}(R)$ is called a normal window whose Fourier Transform satisfies

$\left\{\begin{array}{cc}\hat{w}(\omega)=1, & \text { if } \omega=0 \\ |\hat{w}(\omega)|<1, & \text { if } \omega \neq 0\end{array}\right.$

The local time function $w(t)$ often adopts a normal Gauss window

$w(t)=\frac{1}{\sqrt{2 \pi} \sigma} \exp \left(-\frac{t^{2}}{2 \sigma^{2}}\right)$

where $\sigma>0$. The index $\sigma$ is a vital parameter, which affects the time-frequency resolution of NTFT. We adopt the normal Gauss window and set $\sigma=36$ when plotting the normal time-frequency spectrum of the GPS satellite clock data (Figs. 8, 9, 10, 11).

\section{Inaction method}

To obtain the harmonics and time-varying harmonics directly from the NTFT, Liu and Hsu $(2009,2011)$ and Liu et al. (2016) proposed the inaction method, which is essentially a forthright estimation of the harmonics and timevarying harmonics. Its theoretical derivation is as follows:
Inaction principle A time-harmonic $h(t)$ can be written as

$h(t)=A \exp (i \beta t)=|A| \exp (i(\beta t+\Delta))$

Applying an NTFT $\Psi$ to $h(t)$ yields

(i) $|\Psi h(\tau, \varpi)|=$ Maximum $=|h(\tau)| \Leftrightarrow \varpi=\beta, \forall \tau \in R$

(ii) $\Psi h(\tau, \beta)=h(\tau), \forall \tau \in R$

where $A$ is the complex amplitude, the constant $\beta \in R$ is the angular frequency, constant, $\Delta \in[0,2 \pi)$ is the initial phase, $\beta t+\Delta$ is the immediate (or local) phase, " $\forall$ " means "for any", and " $\Leftrightarrow$ " means "if and only if".

Proof The kernel of the NTFT satisfies

1. $|\hat{\psi}(\omega, \varpi)|=$ Maximum $=1 \Leftrightarrow \omega=\varpi$

Applying an NTFT to $h(t)$, we have

$\Psi h(\tau, \varpi)=\int_{R} A \exp (i \beta t) \bar{\psi}(t-\tau, \varpi) \mathrm{d} t$

If $t-\tau=t^{\prime}, t=t^{\prime}+\tau$, then

$\Psi h(\tau, \varpi)=\int_{R} A \exp \left(i \beta\left(t^{\prime}+\tau\right)\right) \bar{\psi}\left(t^{\prime}, \varpi\right) \mathrm{d} t^{\prime}$

$=A \exp (i \beta \tau) \int_{R} \exp \left(i \beta t^{\prime}\right) \bar{\psi}\left(t^{\prime}, \varpi\right) \mathrm{d} t^{\prime}$

$=h(\tau) \int_{R} \bar{\psi}\left(t^{\prime}, \varpi\right) \exp \left(i \beta t^{\prime}\right) \mathrm{d} t^{\prime}$

$=h(\tau) \overline{\hat{\psi}}(\beta, \varpi)$

Thus

$|\Psi h(\tau, \varpi)|=|h(\tau)||\hat{\Psi}(\beta, \varpi)|=|h(\tau)| \hat{\Psi}(\beta, \varpi) \mid$

Considering (12) and (15), we have that, if and only if $\varpi=\beta,|\Psi h(\tau, \varpi)|=$ Maximum $=|h(\tau)|, \forall \tau \in R$. Thus, the fact (10) is proven. Noting (3) and (14), we have that $\Psi h(\tau, \beta)=h(\tau), \forall \tau \in R$. Thus, the fact (11) is proven.

The result as expressed by (10) and (11) is called the inaction principle of the NTFT. It means the harmonic becomes available automatically from its NTFT without the inverse transform. The IM estimates the harmonics and time-varying harmonics in a time signal according to the inaction principle. Equations (10) and (11) mean that for a complex harmonic, its NTFT along the spectral ridge is itself. For a time-varying harmonic, its NTFT along the spectral ridge 
Fig. 1 Simulated 12- and 6-h terms and their amplitude
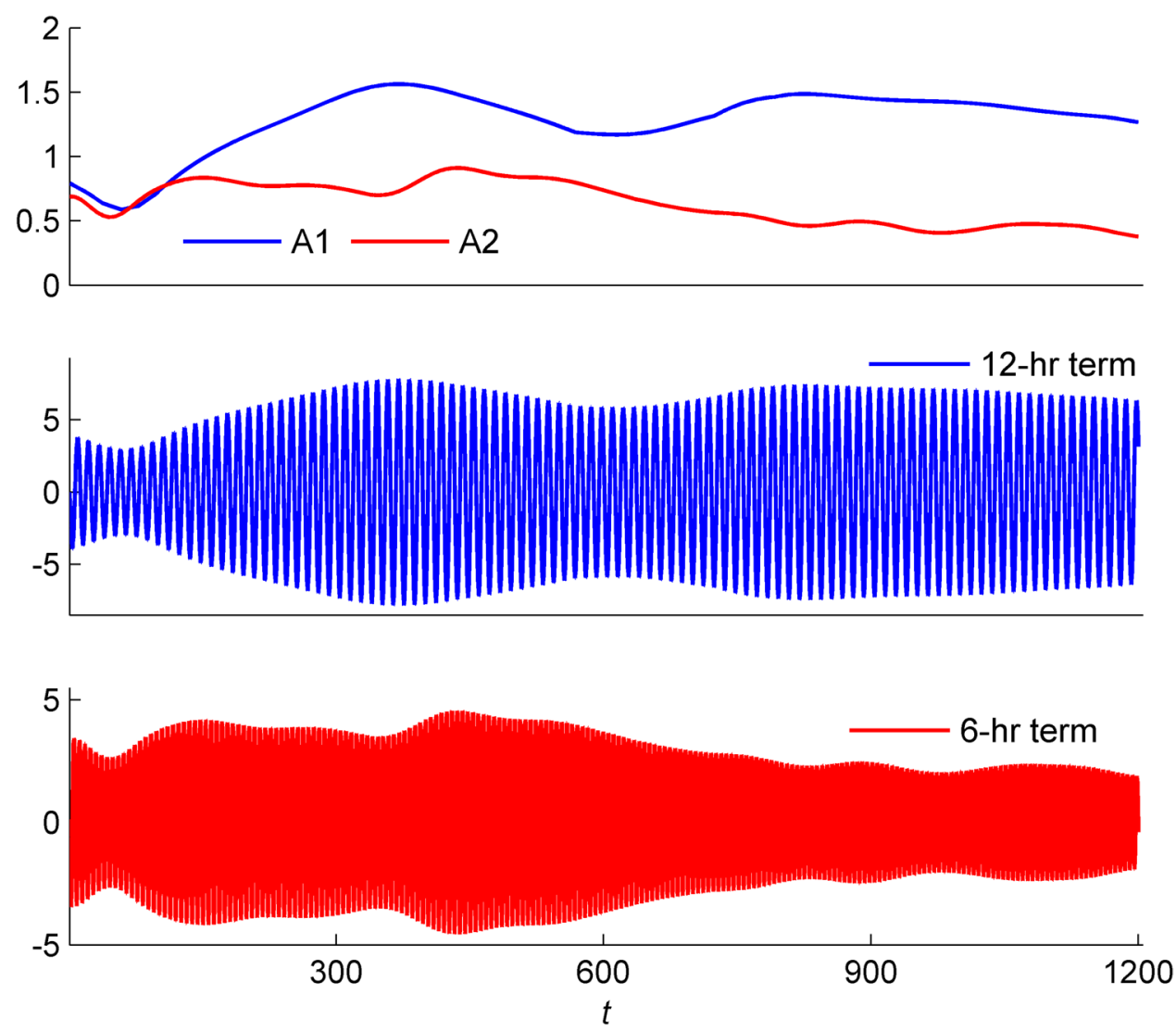

Fig. 2 Simulated noises
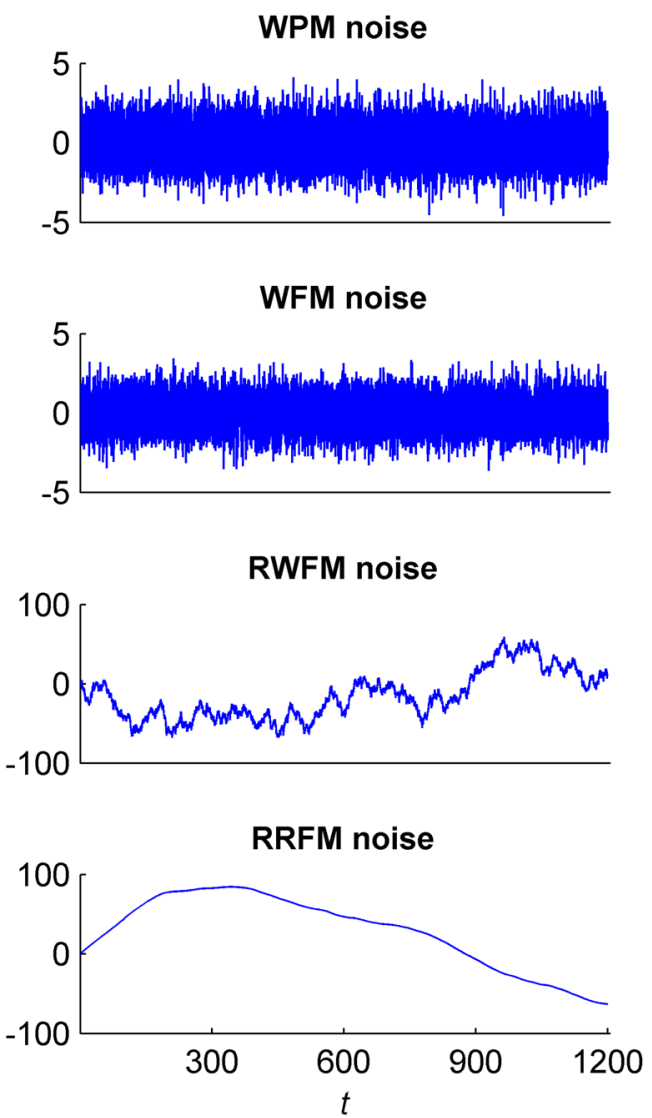

FPM noise

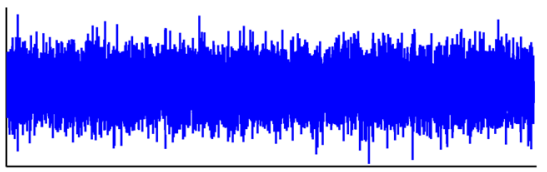

FFM noise

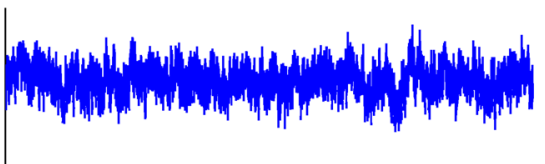

FWFM noise

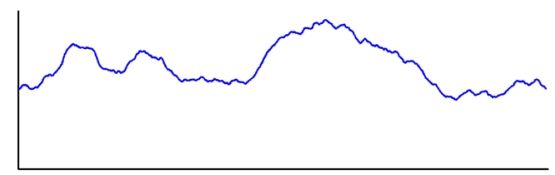

Mixture noise

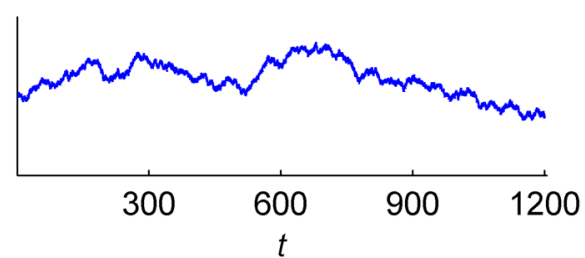


is an estimation or approximation of itself. As a matter of course, for real harmonic or a real time-varying harmonic, the twofold of the real part of its NTFT along the spectral ridge is itself or estimation of itself. On the time-frequency plane of the NTFT spectrum, the minimum zones are applied in the estimation of the harmonics and time-varying harmonics. Relating the band-pass filtering, the IM is essentially line-pass filtering. Thus, the IM can avoid being polluted by noises to the maximum extent and then have the ability to extract the time-varying harmonics with robustness; thus,

\section{Simulation test}

The methods used most often in extracting the harmonics and time-varying harmonics are SSA and ZPDF (Höpfner 2004). The details of SSA and ZPDF can be found in Chen et al. (2013) and Gustafsson (1996), respectively. To compare the extraction accuracy of these two filtering methods with IM, we simulate a set of time series which contain two time-varying harmonics and various noises. The sampling interval is $1 / 12$,

$y(t)=5 A_{1}(t) \cos \left(\frac{2 \pi t}{12}+\frac{\pi}{3}\right)+5 A_{2}(t) \cos \left(\frac{2 \pi t(1+0.00006(t-600))}{6}+\frac{\pi}{6}\right)+$ noises $0 \leq t \leq 1200$

IM is very suitable to extract the time-varying harmonics from the mixed signals, especially when it includes a lot of noise components. In this case, extracting time-varying harmonic terms using any type of band-pass filtering will be inevitably polluted by noises. where the first and second terms on the right-hand side of the equation are two original sub-signals, namely $12-$ and 6-h terms, respectively, which are shown in Fig. 1 (middle and bottom panels); the time-varying amplitudes of these two original sub-signals are $A_{1}(t)$ and $A_{2}(t)$ and can be seen in the top panel. It should be noted that the 6-h term has timevarying frequencies. The third term contains various noises, such as White phase modulation (WPM) noise, Flicker phase modulation (FPM) noise, White frequency modulation (WFM) noise, Flicker frequency modulation (FFM) noise,
Fig. 3 Synthesized signals containing the same 12- and 6-h terms and various noises, respectively. The component in the bracket is SNR of the synthesized signals with a different value

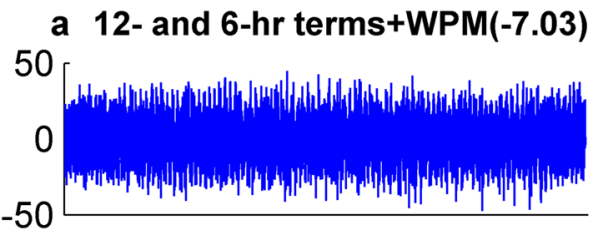

c 12- and 6-hr terms+WFM(-5.74)

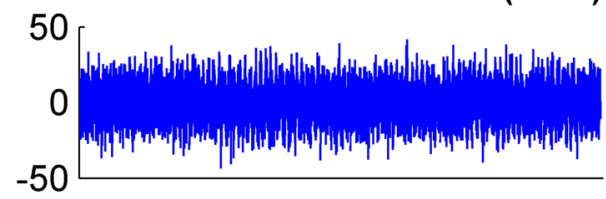

e 12- and 6-hr terms+RWFM(-16.39)

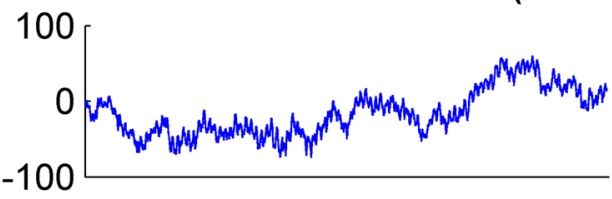

g 12- and 6-hr terms+RRFM(-20.29)

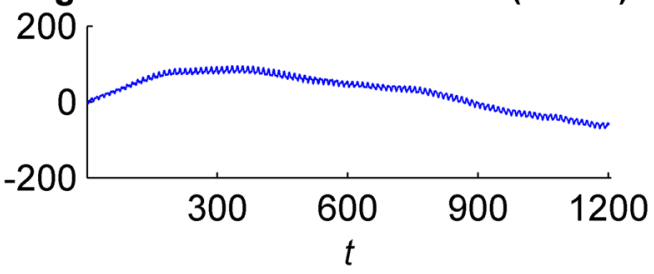

b 12- and 6-hr terms+FPM(-7.14)

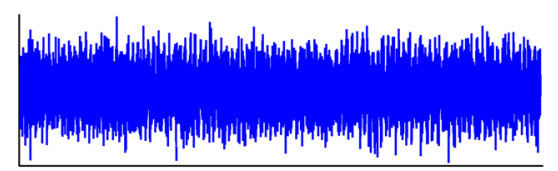

d 12- and 6-hr terms+FFM(-5.26)

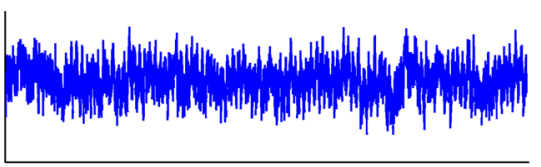

f 12- and 6-hr terms+FWFM(-17.97)

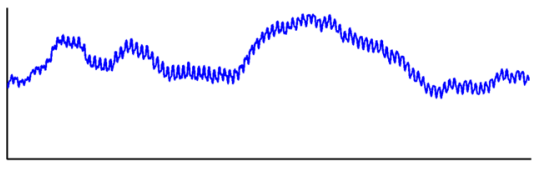

h 12- and 6-hr terms+Mixture(-21.75)

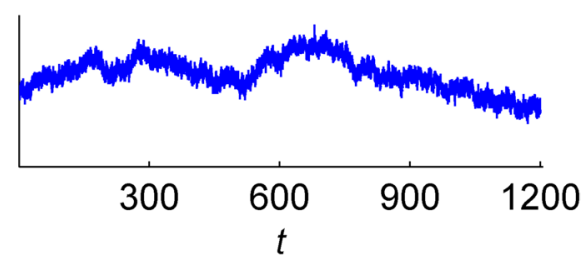


Random Walk frequency modulation (RWFM) noise, Flicker Walk frequency modulation (FWFM) noise, Random Run frequency modulation (RRFM) noise, and mixture noise, which are shown in Fig. 2, respectively.

To compare the robustness of these three methods regarding extracting the harmonics and time-varying harmonics, we simulated the synthesized signals with different signalto-noise ratio (SNR). As shown in Fig. 3, the synthesized signals contain 12- and 6-h terms, and various types of noises.

As shown in Fig. 3a, b, c, d, h, the simulated 12- and 6-h terms are almost covered by the noise. The extracted terms by these three methods can be seen in Figs. 4, 5, 6 and 7. Rows from top to bottom correspond to different types of noise, and the method adopted is labeled on each figure. In these figures, the blue and black lines indicate the extracted terms and the error between the true harmonics and the extracted terms, respectively.

As shown in Fig. $4 \mathrm{a}-\mathrm{i}$, the graphics of 12 -h terms extracted by these three methods all seem to be similar when processing the synthesized signals, which includes White PM, Flicker PM and White FM, respectively. In fact, in this case, the extraction precision of IM is slightly better than SSA and ZPDF. The results of statistical error and correlation coefficient can be seen in Tables 2 and 3, respectively.
As shown in Fig. $4 j-1$, the graphics of the 12 -h terms extracted by SSA and ZPDF are apparently distorted when processing the Flicker FM noise. In this case, we see that the 12-h term extracted by IM is less affected by the Flicker PM noise compared to the methods SSA and ZPDF, which can be proven to be the results of statistical error (Table 2) and correlation coefficient (Table 3).

As shown in Fig. 5a, j, the burr appeared in the extracted 12-h terms by SSA, which includes the Random Walk FM and the mixed noise, respectively. The 12-h terms extracted by ZPDF are apparently irregular as shown in Fig. 5b, k; however, in this case, the 12-h term extracted by IM is the smoothest among these three methods. In this case, it indicates that the 12 -h harmonic is more accurately recovered by the IM than both the SSA and ZPDF when the signal includes Random Walk FM and mixed noise (as shown in Tables 2, 3).

As shown in the first row of Fig. 6, the errors of the extracted 6-h terms by ZPDF and IM are significantly less than by SSA when processing the synthesized signals, which include the White PM noise. The results are shown in Tables 2 and 3. The 6-h terms extracted by SSA and ZPDF are slightly distorted as shown in Fig. $6 \mathrm{~g}, \mathrm{~h}, \mathrm{j}, \mathrm{k}$. In the case, the 6-h term extracted by IM is the smoothest among these three methods. It indicates that the 6-h term
Fig. 4 Extracted 12-h terms (blue) and errors (red) in the case of the signals polluted by different types of noises. Rows from top to bottom correspond to the White PM noise, the Flicker PM noise, the White FM noise and the Flicker FM noise
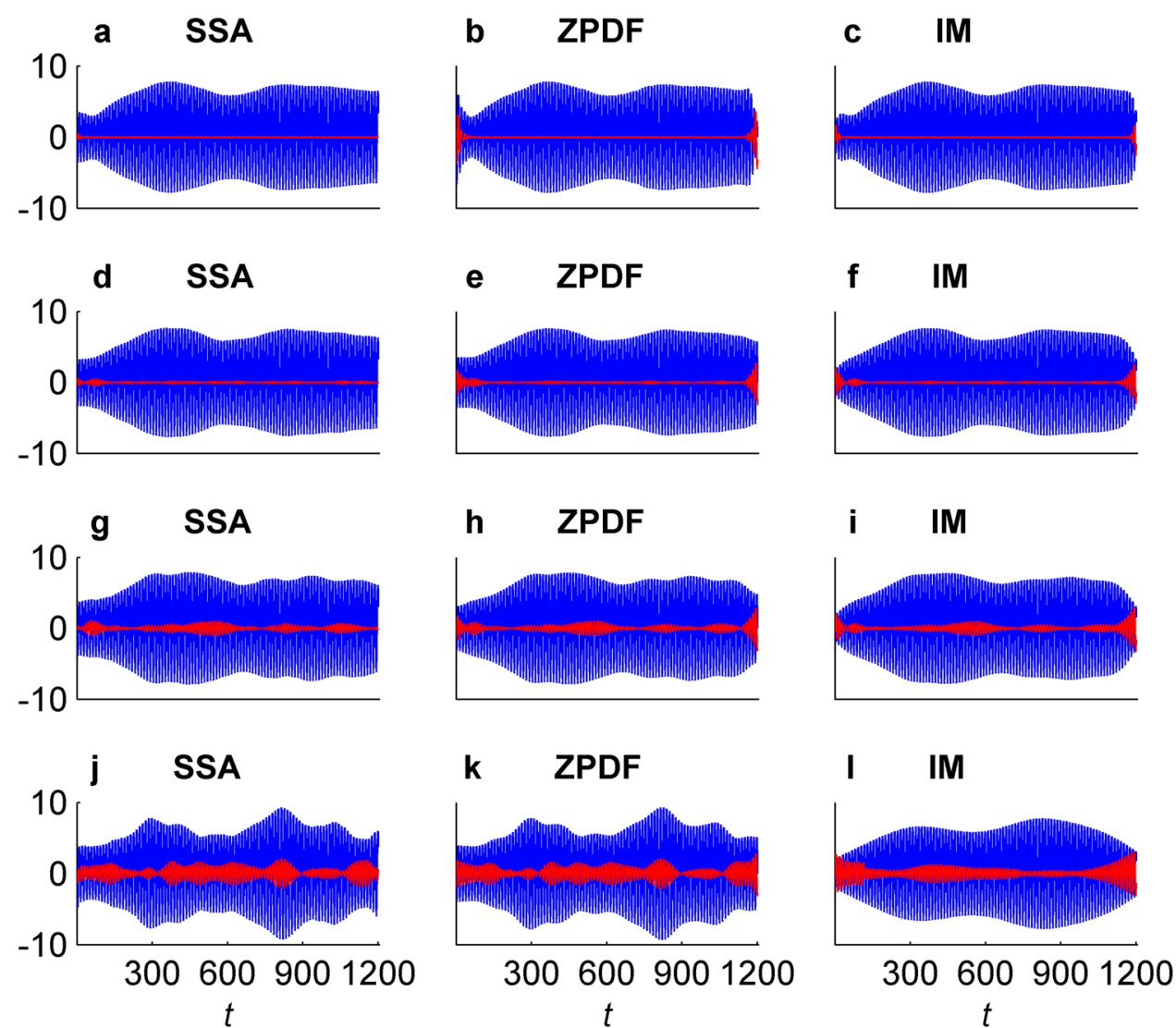
extracted by IM is less affected by the White FM noise and the Flicker FM noise than those by SSA and ZPDF.

As shown in Fig. 7, top and bottom rows, the 6-h terms extracted by SSA and ZPDF are slightly distorted. In the case, the 6-h term extracted by IM is smoothest among these three methods. It indicates that the 6-h term extracted by IM is less affected by the Random Walk FM noise and the mixed noise than the terms extracted by SSA and ZPDF.

For evaluating the extraction accuracy of these three methods, the relative error was introduced as follow:

Relative error $=\frac{\text { RMSE }}{\text { signal's RMS }}=\frac{\sqrt{\sum_{i=1}^{N}\left(x_{i}-\hat{x}_{i}\right)^{2} / N}}{\sqrt{\sum_{i=1}^{N} x_{i}^{2} / N}}$,

where $x_{i}$ and $\hat{x}_{i}$ indicate the $i$ th simulation value and $i$ th estimation value, respectively. $N$ is the length of the simulation data.

The correlation coefficient and relative errors can be seen in Tables 2 and 3, respectively. Because of the edge effect present in all of these three methods, some values at both ends of extraction time series are removed when evaluating the extraction accuracy.

As shown in Table 2, the correlation coefficient between the 12-h terms extracted by IM and the simulation harmonics are all greater than 0.99. As shown in Tables 2 and 3, the correlation coefficient between the extracted terms by IM and the simulation harmonics, and the accuracy of extraction by IM are all the best, except in the case of the Random Run FM only. This simulation demonstrates that the IM can robustly extract time-varying harmonics from a noisy time signal, even if the SNR is -21 .

\section{Time-frequency analysis of the GPS satellite clocks}

To study the time-frequency characteristic of the GPS satellite clocks, the IGS Final $300 \mathrm{~s}$ satellite clocks as shown in Table 1 are adopted for the time May 8-July 2, 2016. The GPS satellite clocks with significant periodicity are chosen to analysis their time-frequency characteristic. In addition, the two GPS cesium clock, prn08 and 24, are also chosen for comparison. To reduce the effect of the trend term on the time-frequency spectrum as far as possible, quartic polynomials are used for removing the trend term of the GPS
Fig. 5 Extracted 12-h terms (blue) and errors (red) in the case of the signals polluted by different types of noises. Rows from top to bottom correspond to the Random Walk FM noise, the Flicker Walk FM noise, the Random FM noise and the mixed noise
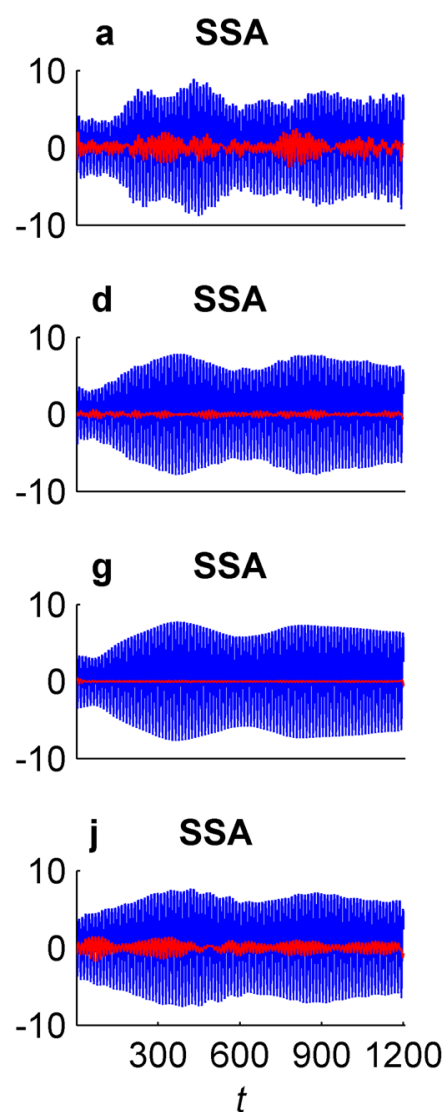
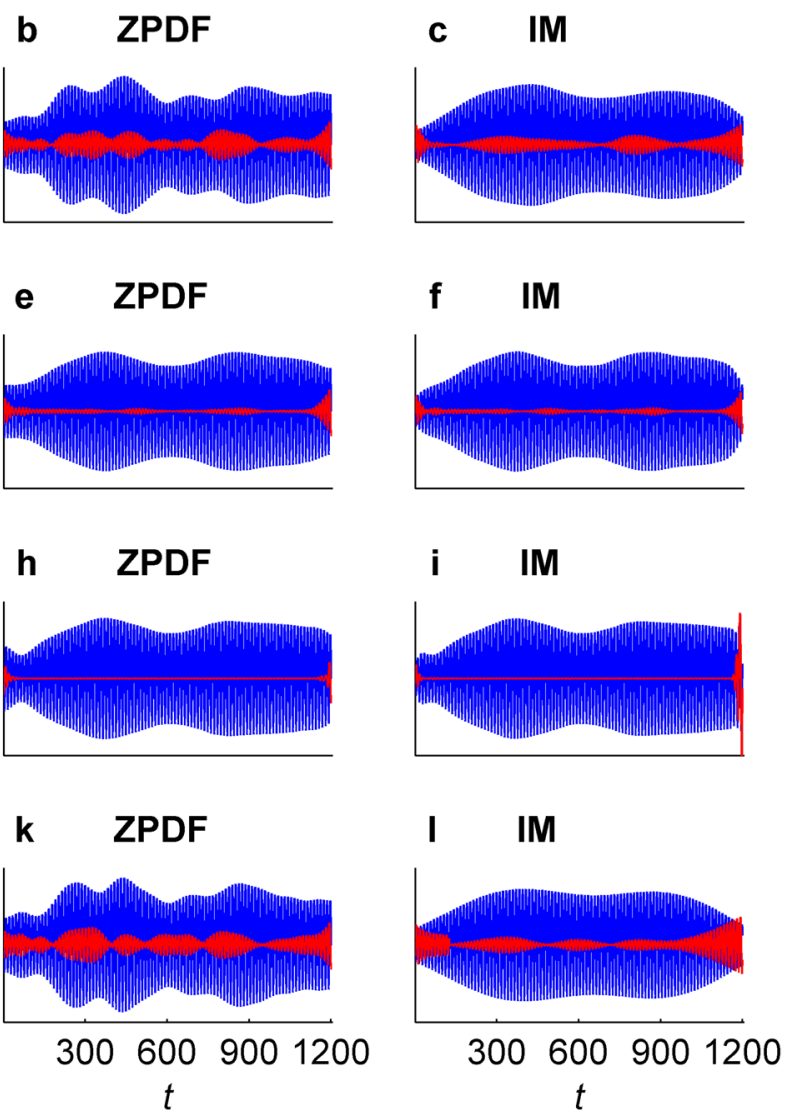
Fig. 6 Extracted 6-h terms (blue) and errors (red) in the case of the signals polluted by different types of noises. Rows from top to bottom correspond to the White PM noise, the Flicker PM noise, the White FM noise and the Flicker FM noise
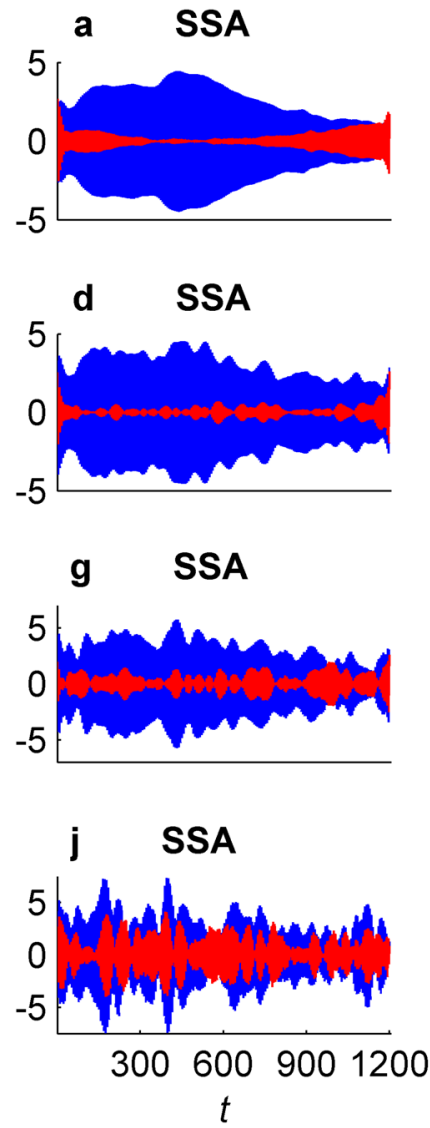
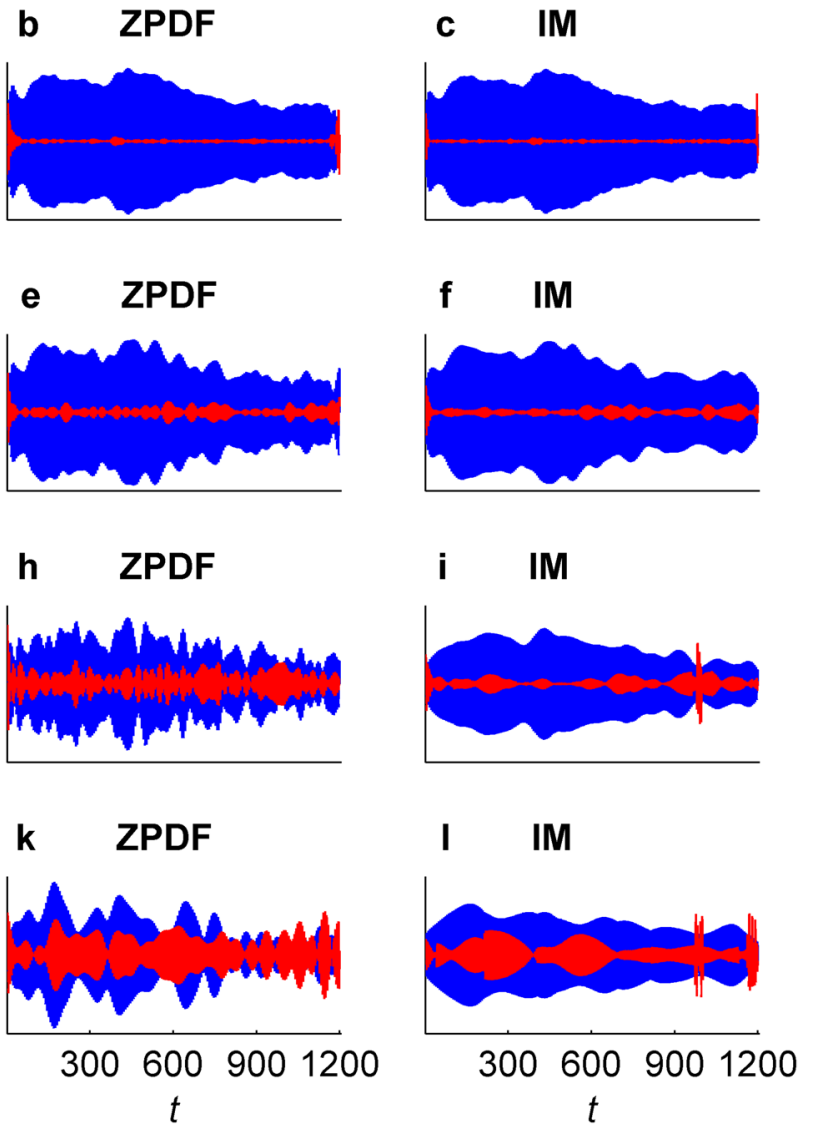

satellite clocks. According to the normal wavelet transform (8), the NTFT spectrum of the GPS satellite clocks can be obtained.

As can be seen in Figs. 8, 9 and 10, significant 6- or 12-h periodic signals exist in GPS rubidium clocks; most of the rubidium atomic clock contains both of the two periodic signals, especially, the block IIF rubidium atomic clock. Even some satellite clocks contain 3- and 4-h periodic signals, such as prn06 and prn21. As can be seen in Fig. 10, the GPS cesium clocks, prn08 and 24, do not contain these two periodic signals. It is possible that the short term of the GPS cesium clocks is worse than that of the rubidium clock (Cernigliaro et al. 2013a, b); in that case the periodic signal may be drowned by the noise of the GPS cesium clocks. These conclusions from above agree with Senior (2008).

\section{Amplitude variations of the harmonic}

As can been seen in Figs. 8, 9 and 10, there are significant 6- and 12-h periodic terms are both of these satellite clocks, such as prn01, 03, 05, 06, 09, 12, 13, 15, 16, 17, 18, 21, 22, $25,26,27,29,30,31$ and 32 . Then, according to the inaction method, equations 7 and 8 , the 6 - and 12 -h periodic signals of these satellite clocks can be extracted from their NTFT spectrum, respectively.

As can be seen in Fig. 11, the amplitudes of the 6-h periodic terms of these satellite clocks show different variations. For example, within this time span, prn12, 13, 16 have a gradually decreased fluctuation, whereas prn21, 25, 26, 31 have a gradually increased fluctuation. In addition, the amplitudes of the 6-h terms of prn16, 17, 29 and 31 are relatively large, being greater than $0.2 \mathrm{~ns}$.

As can be seen in Fig. 12, the amplitudes of the 12-h periodic terms of these satellite clocks show significant variations, and amplitudes of the 12-h terms of prn05, 12, 13, $16,17,18,27,29,30$, and 31 are relatively large, being greater than $0.2 \mathrm{~ns}$.

As shown in Figs. 11 and 12, the amplitudes of the 12-h terms are generally greater than the 6 -h terms. Figures 11 and 12 both show that their amplitudes of the 6- and 12-h terms vary over time, which should be taken into full consideration when modeling the GPS satellite clocks. In addition, we note that the amplitudes at the beginning and end of the 6- and 12-h terms may be less accurate because of an edge effect. 
Fig. 7 Extracted 6-h terms (blue) and errors (red) in the case of the signals polluted by different types of noises. Rows from top to bottom correspond to Random Walk FM noise, Flicker Walk FM noise, Random FM noise, and the mixed noise

Table 2 Correlation coefficient between the estimation/ extraction or the simulation harmonics
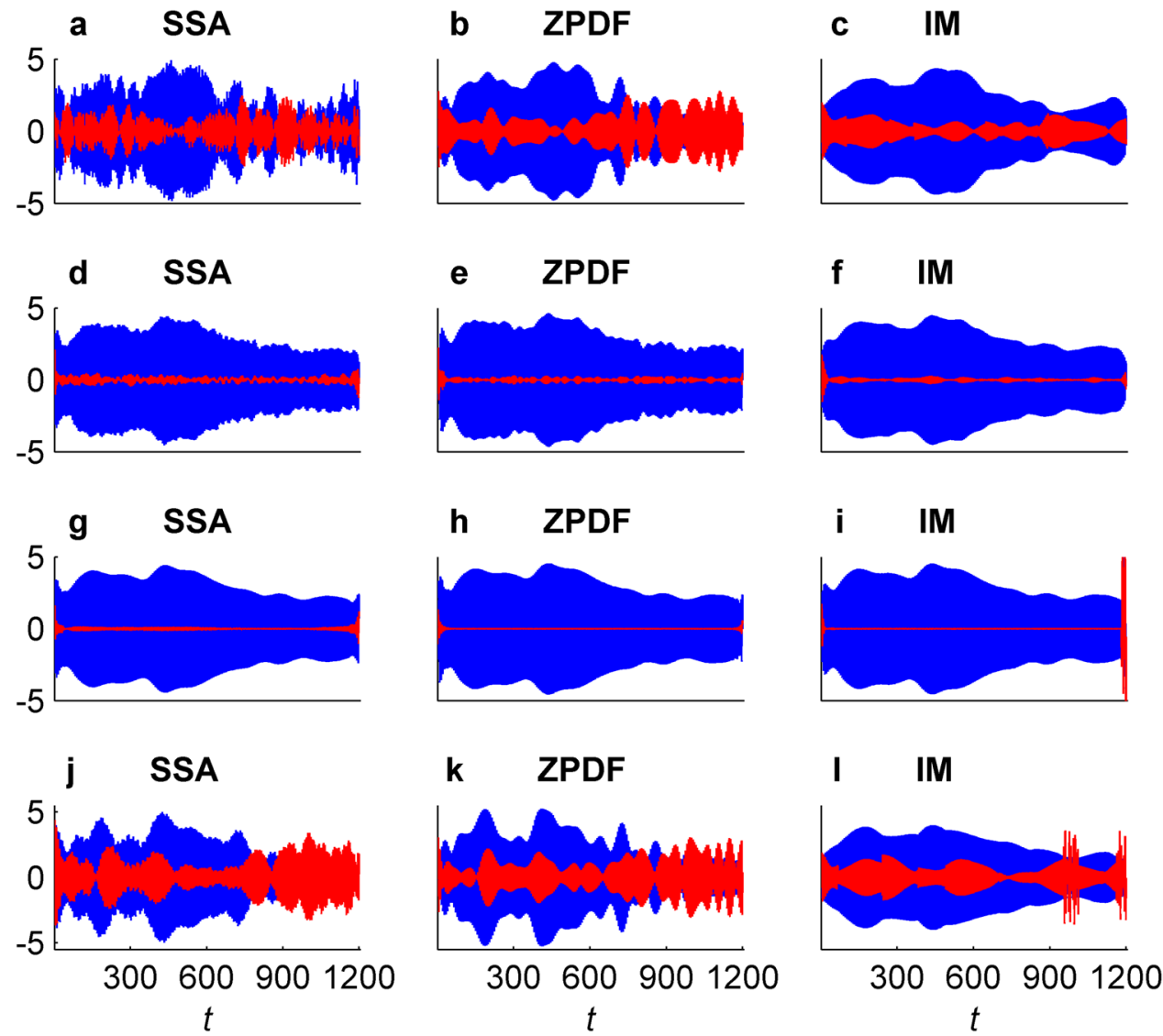

\section{Comparison of the Hadamard deviation}

To verify whether the 6- and 12-h terms are extracted completely and exactly from GPS satellite clock using the IM, the Hadamard deviation of the satellite clocks before and after extracting these periodic terms are compared. More information on the Hadamard deviation can be found in Hutsell (1996) and references therein.
As shown in Fig. 13, after removing the 6- and 12-h periodic terms, the oscillation phenomenon in Hadamard deviation virtually disappears, which demonstrate that these periodic signals have been extracted effectively. In addition, with respect to the red line, the blue line indicates that the Hadamard deviation of the satellite clocks within the averaging interval 300-3000s is the response of satellite clock noise, for averaging intervals beyond 3000 s, it is the response of 
Table 3 Relative errors of the estimation or extraction using the three methods

\begin{tabular}{|c|c|c|c|c|c|c|c|c|}
\hline \multirow[t]{3}{*}{ No. } & \multirow[t]{3}{*}{ Noise type } & \multirow[t]{3}{*}{ SNR } & \multicolumn{6}{|c|}{ Relative error (percentage) } \\
\hline & & & \multicolumn{3}{|c|}{ 12-h term } & \multicolumn{3}{|c|}{ 6-h term } \\
\hline & & & SSA & ZPDF & IM & SSA & ZPDF & IM \\
\hline 1 & White PM & -7.03 & 0.63 & 0.90 & 0.47 & 15.66 & 3.05 & 2.64 \\
\hline 2 & Flicker PM & -7.14 & 2.68 & 2.85 & 2.65 & 10.03 & 10.34 & 7.86 \\
\hline 3 & White FM & -5.74 & 8.68 & 8.33 & 8.01 & 27.73 & 33.98 & 18.88 \\
\hline 4 & Flicker FM & -5.26 & 13.01 & 17.35 & 12.26 & 55.96 & 57.32 & 41.28 \\
\hline 5 & Random walk FM & -16.39 & 19.00 & 18.13 & 10.96 & 35.62 & 39.46 & 23.71 \\
\hline 6 & Flicker walk FM & -17.97 & 5.16 & 4.28 & 4.10 & 6.99 & 4.99 & 3.78 \\
\hline 7 & Random run FM & -20.29 & 1.19 & 0.47 & 0.37 & 2.79 & 0.32 & 0.44 \\
\hline 8 & Mixture & -21.75 & 12.24 & 19.43 & 11.10 & 48.31 & 45.13 & 34.35 \\
\hline
\end{tabular}
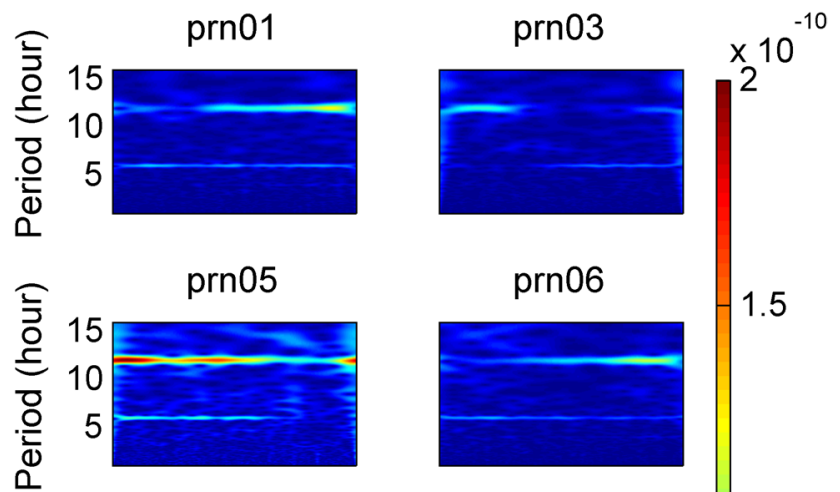

1.5
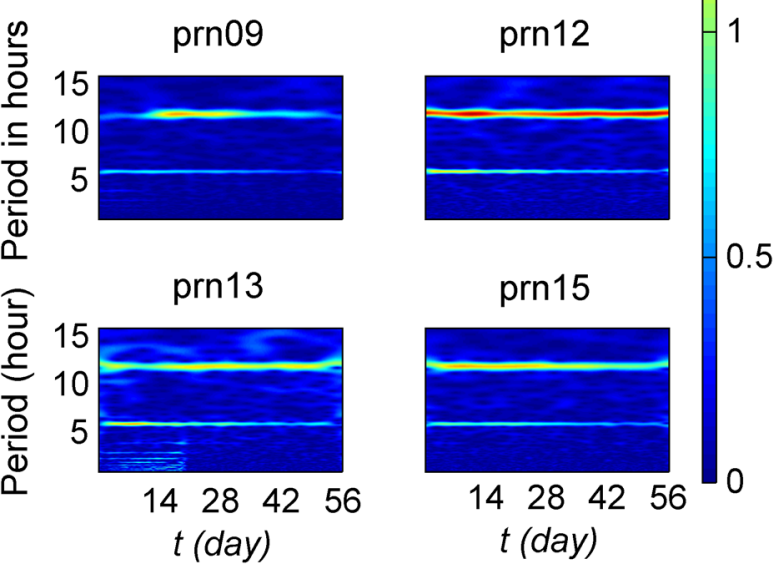

Fig. 8 NTFT spectrum of GPS satellite clocks (prn01, 03, 05, 06, 09, $12,13$ and 15$)$

periodic signals existing in satellite clocks. The red lines demonstrate that the Hadamard deviation of the remaining signal after removing the periodic terms from satellite clocks is the real response of the clock noise within the averaging
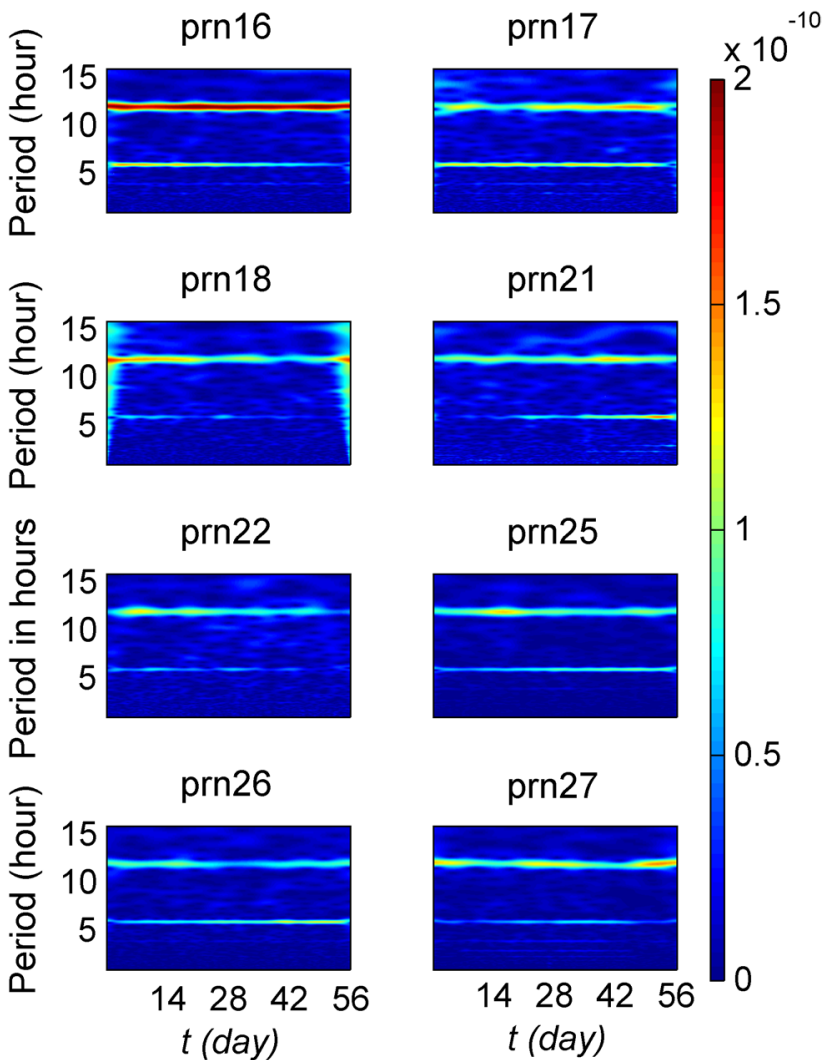

0.5

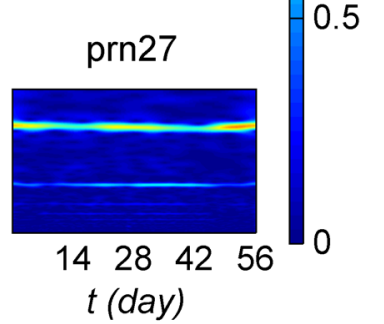

Fig. 9 NTFT spectrum of GPS satellite clocks (prn16, 17, 18, 21, 22, 25,26 and 27)

interval 90000s. Therefore, to evaluate the clock noise characteristic within the long-term averaging interval, it is possible to extract the periodic terms using the IM. 


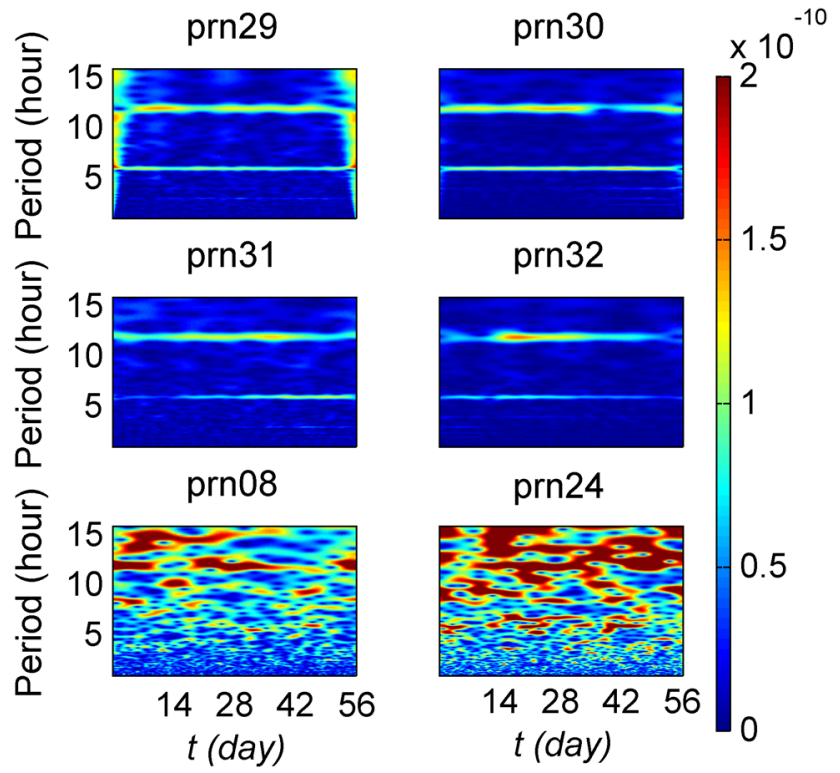

Fig. 10 NTFT spectrum of GPS satellite clocks (prn29, 30, 31, 32, 08 , and 24)

\section{Conclusions}

We demonstrated the performance and robustness of the IM, which essentially is line-pass filtering, on extracting the harmonics and time-varying harmonics from a noisy signal. The 12- and 6-h periodic terms in most GPS rubidium clocks have been detected in NTFT spectrums, which can provide an unbiased measurement of the instantaneous frequency, phase and amplitude of the periodic signals. Through analyzing the 6 - and 12 -h periodic terms, we find that their amplitudes show significant variations between May 8 and July 2, 2016, which are very important to model satellite clocks. After removing 6- and 12-h periodic terms, the oscillation phenomenon in Hadamard deviation virtually disappears, this also shows that these terms have been extracted effectively by IM. Therefore, when evaluating the response of noise within long-term averaging interval using Hadamard deviation, one can detect the periodic signals by NTFT and remove these by the IM.
Fig. 11 6-h periodic terms extracted by IM from GPS satellite clock bias
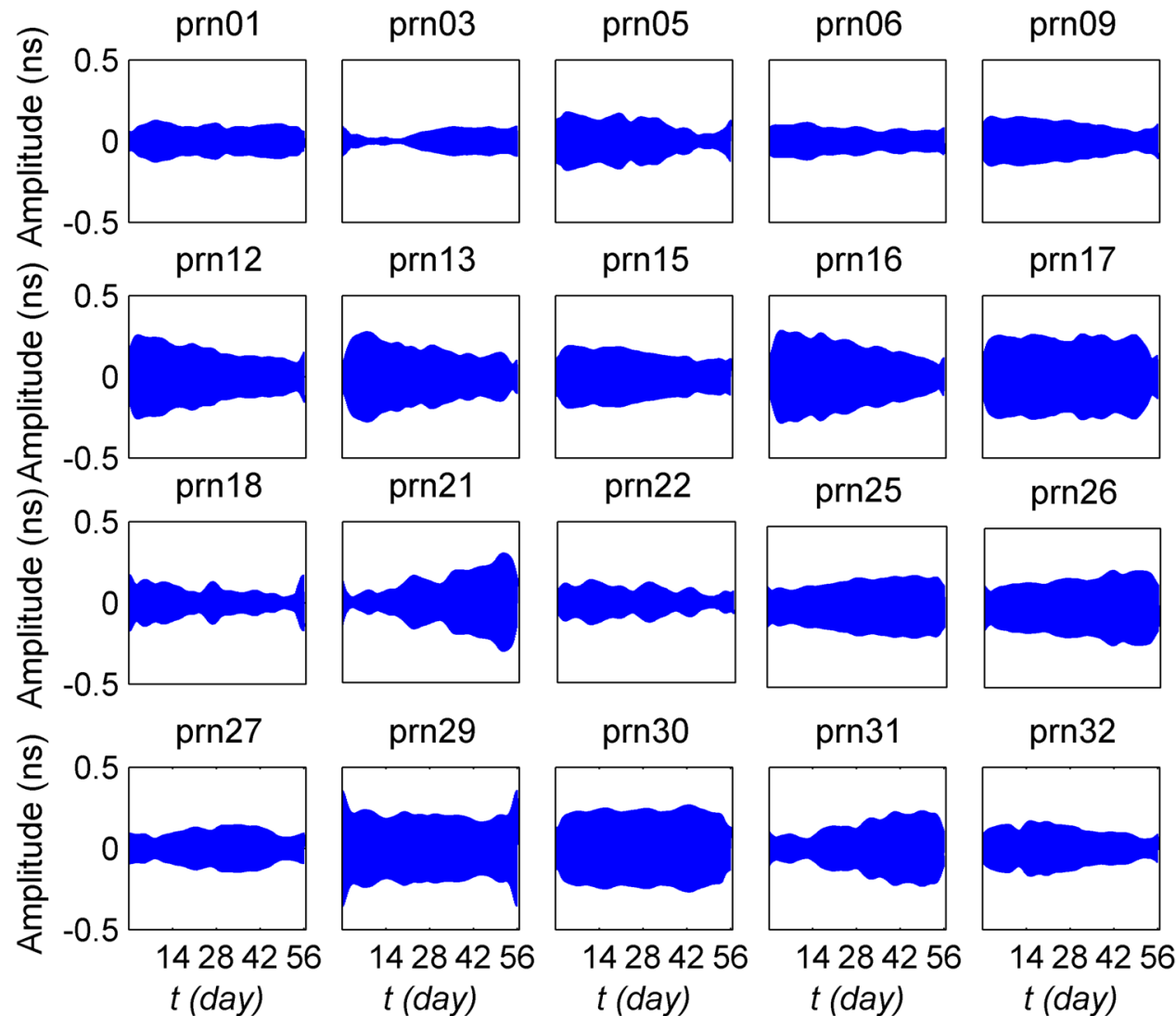

prn17
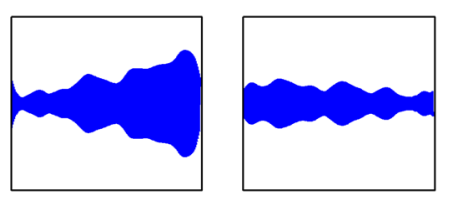

prn25

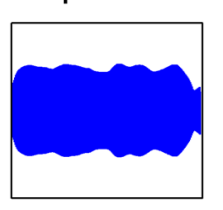

prn26
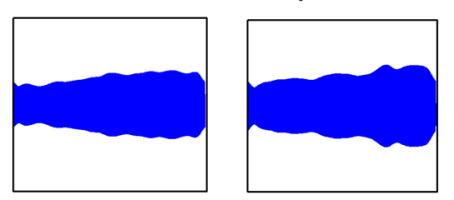

prn29

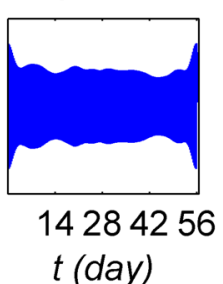

prn30

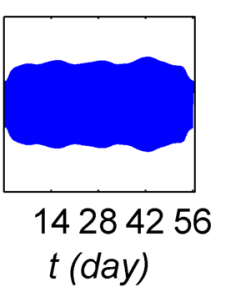

prn31

prn32
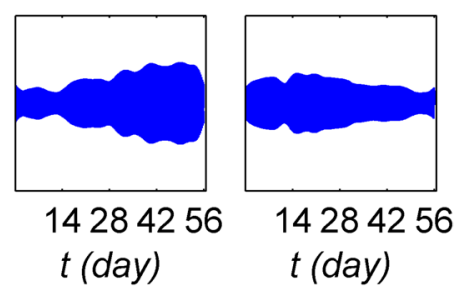
Fig. 12 12-h periodic terms extracted by IM from GPS satellite clock bias
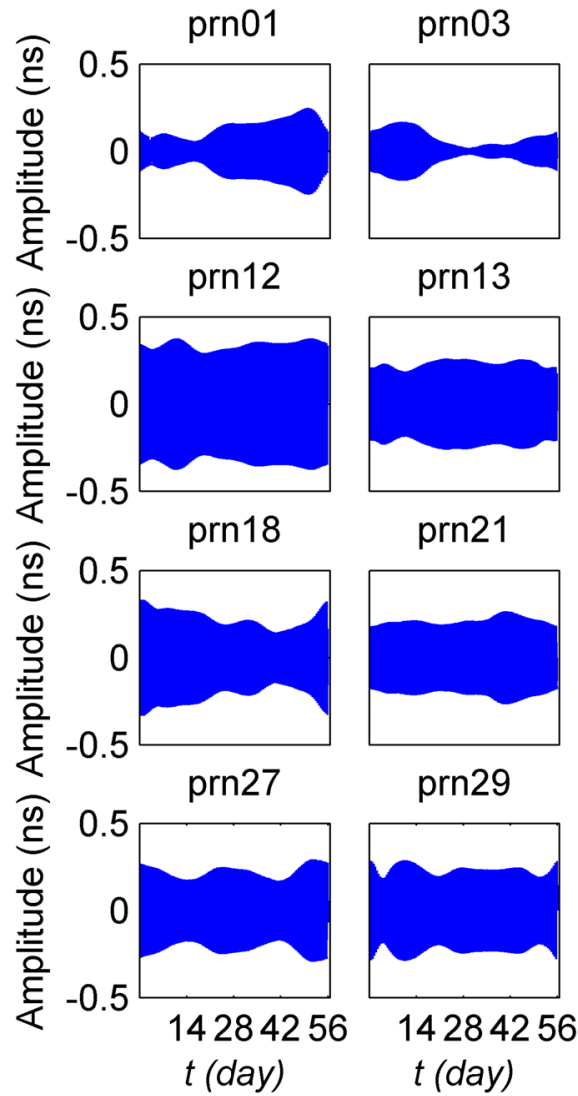

prn13

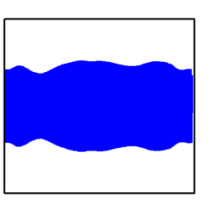

prn21

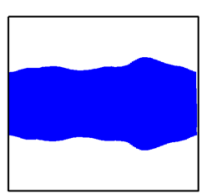

prn29

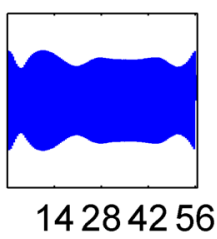

$t$ (day)

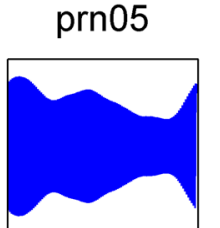

prn15

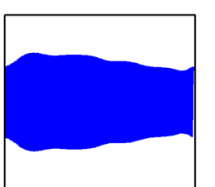

prn22

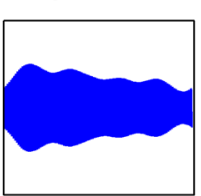

prn30

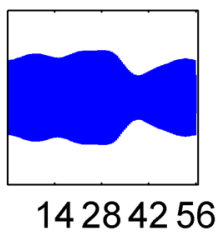

$t$ (day)

14284256

$t$ (day)

$t$ (day)

prn32
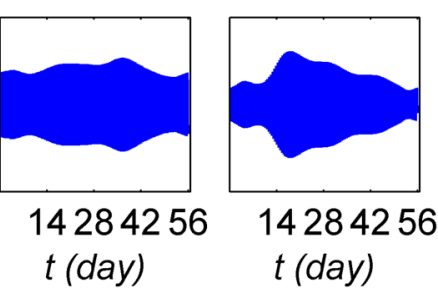

Fig. 13 Comparison of the Hadamard deviation. AI is an abbreviation of averaging interval. The blue and red lines indicate the Hadamard deviation of GPS satellite clocks and the Hadamard deviation of the residual after removing the 6- and 12-h periodic terms, respectively

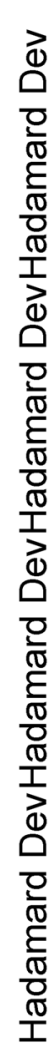

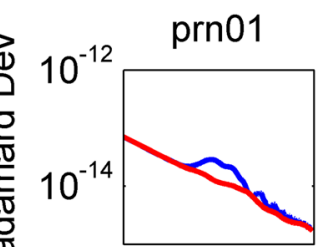

prn03

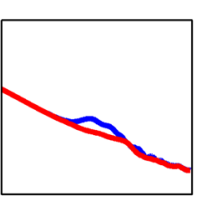

prn13

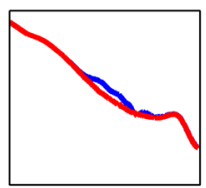

prn21

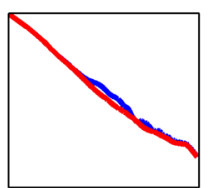

prn29
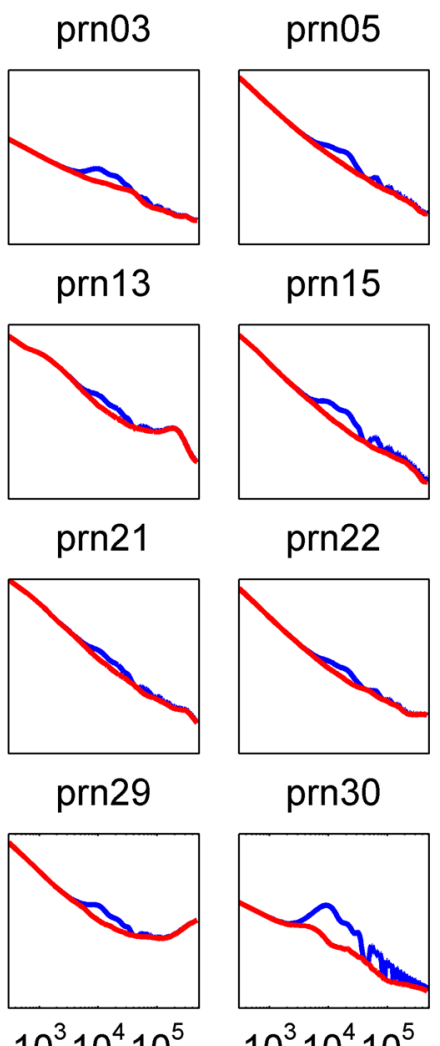

prn22

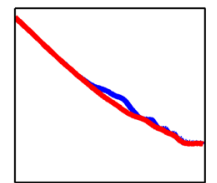

prn30

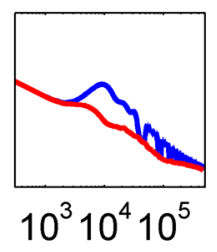

$\mathrm{Al}$ (s)

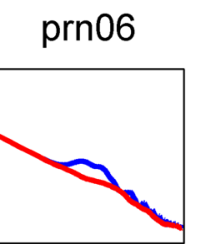

prn16

prn09

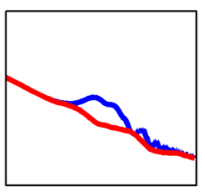

prn17

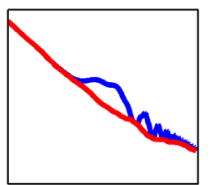

prn25

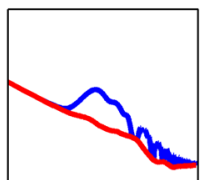

prn31

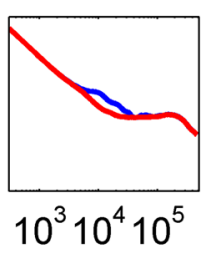

$\mathrm{Al}$ (s)

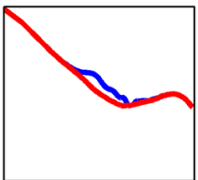

prn26

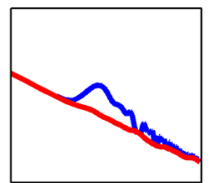

prn32

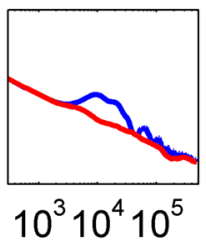

$\mathrm{Al}$ (s) 
Acknowledgements We are grateful to the International Global Navigation Satellite Systems Service (IGS) for providing the GPS clock data. This study was supported by the National Natural Science Foundation of China (Grant 41074050) and Ministry of Science and Technology of the People's Republic of China (Grant 2011YQ120045). In addition, the anonymous reviewers are thanked for their remarks and suggestions which were helpful in preparing the revised version.

Open Access This article is distributed under the terms of the Creative Commons Attribution 4.0 International License (http://creativeco mmons.org/licenses/by/4.0/), which permits unrestricted use, distribution, and reproduction in any medium, provided you give appropriate credit to the original author(s) and the source, provide a link to the Creative Commons license, and indicate if changes were made.

\section{References}

Amin MG, Borio D, Zhang YD, Galleani L (2017) Time-frequency analysis for GNSSs: from interference mitigation to system monitoring. IEEE Signal Process Mag 34(5):85-95

Cernigliaro A, Valloreia S, Galleani L, Tavella P (2013a) GNSS space clocks: performance analysis. In: Proceedings of the international conference on localization and GNSS (ICL-GNSS), IEEE, Turin, Italy, pp 1-5. https://doi.org/10.1109/ICL-GNSS.2013.6577251

Cernigliaro A, Valloreia S, Fantino G, Galleani L, Tavella P (2013b) Analysis on GNSS space clocks performances. In: European frequency and time forum \& international frequency control symposium (EFTF/IFC), IEEE, Prague, Czech Republic, pp 835-837

Chao BF, Chung W, Shih Z, Hsieh Y (2014) Earth's rotation variations: a wavelet analysis. Terra Nova 26(4):260-264

Chen Q, van Dam T, Sneeuw N, Collilieux X, Weigelt M, Rebischung P (2013) Singular spectrum analysis for modeling seasonal signals from GPS time series. J Geodyn 72:25-35

Galleani L (2008) Detection of changes in clock noise using the timefrequency spectrum. Metrologia 45(6):143-153

Galleani L, Tavella P (2016) The dynamic allan variance V: recent advances in dynamic stability analysis. IEEE Trans Ultrason Ferroelectr Freq Control 63(4):624-635

Gonzalez F, Cernigliaro A, Tavella P, Boyero JP (2010) Clock strategy experimentation with GIOVE clocks. In: Proceedings of EFTF2010 24th European frequency and time forum, pp 1-8

Gonzalez Martinez FJ (2013) Performance of new GNSS satellite clocks. KIT Scientific, Karlsruhe

Gustafsson F (1996) Determining the initial states in forward-backward filtering. IEEE Trans Signal Process 44(4):988-992

Hauschild A, Montenbruck O, Steigenberger P (2013) Short-term analysis of GNSS clocks. GPS Solut 17(3):295-307

Heo YJ, Cho J, Heo MB (2010) Improving prediction accuracy of GPS satellite clocks with periodic variation behaviour. Meas Sci Technol 21(7):073001

Höpfner J (2004) Low-frequency variations, Chandler and annual wobbles of polar motion as observed over one century. Surv Geophys 25(1):1-54

Hutsell S (1996) Relating the Hadamard variance to MCS Kalman filter clock estimation. In: 27th annual precise time and time interval (PTTI) applications and planning meeting, San Diego, USA, pp 291-301

Liu L, Hsu H (2009) Time-frequency transform: inversion and normalization. Hubei Science and Technology Press, Hubei
Liu L, Hsu H (2011) Inversion and normalization of time-frequency transform. In International conference on multimedia technology (ICMT), pp 2164-2168

Liu L, Hsu H, Grafarend EW (2007) Normal Morlet wavelet transform and its application to the Earth's polar motion. J Geophys Res Solid Earth 112(B08401):1-14

Liu L, Su X, Wang G (2016) Discussion on normal time-frequency transform. J Navig Position 4(4):1-5. https://doi.org/10.16547 /j.cnki.10-1096.20160401

Marquis WA, Reigh DL (2015) The GPS block IIR and IIR-M broadcast L-band antenna panel: its pattern and performance. Navigation 62(4):329-347

Montenbruck O, Steigenberger P, Schoenemann E, Hauschild A, Hugentobler U, Dach R, Becker M (2012) Flight characterization of new generation GNSS satellite locks. Navigation 59(4):291-302

Popinski W, Kosek W (1995) The Fourier transform band pass filter and its application for polar motion analysis. Artif Satell Planet Geodesy 30(1):9-25

Senior KL, Ray JR, Beard RL (2008) Characterization of periodic variations in the GPS satellite clocks. GPS Solut 12(3):211-225

Sesia I, Galleani L, Tavella P (2011) Application of the dynamic Allan variance for the characterization of space clock behavior. IEEE Trans Aerosp Electron Syst 47(2):884-895

Smylie DE, Henderson GA, Zuberi M (2015) Modern observations of the effect of earthquakes on the Chandler wobble. J Geodyn 83:85-91

Swift ER, Hermann BR (1988) Orbit period frequency variations in the GPS satellite clocks. In: Proceedings of the 20th annual precise time and time interval (PTTI) applications and planning meeting, Vienna, VA (USA), Nov 29-Dec 1, pp 87-100

Vannicola F, Beard R, White J, Senior K, Largay M, Buisson JA (2010) GPS block IIF atomic frequency standard analysis. In: Proceedings of the 42 nd annual precise time and time interval (PTTI) application and planning meeting, Reston, VA, Canada, pp 181-195

Weiss M, Shome P, Beard R (2010) On-board GPS clock monitoring for signal integrity. In: Proceedings of the 42th annual precise time and time interval (PTTI) applications and planning meeting, Virginia, USA, pp 465-479

Wu Z, Huang NE (2009) Ensemble empirical mode decomposition: a noise-assisted data analysis method. Adv Adapt Data Anal 1(1): $1-41$

Zhou S, Hu X, Wu B, Liu L, Qu W, Guo R, Shi X (2011) Orbit determination and time synchronization for a GEO/IGSO satellite navigation constellation with regional tracking network. Sci China Phys Mech Astron 54(6):1089-1097

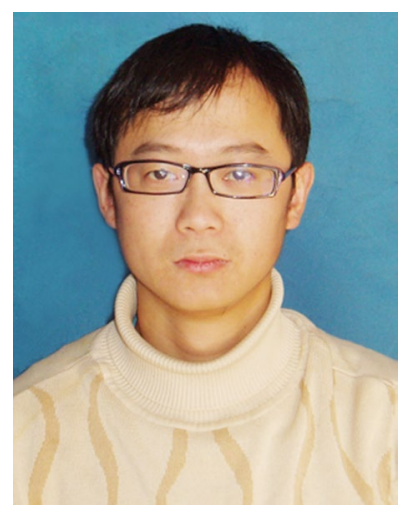

Guocheng Wang received the Ph.D. degree of geodesy from University of Chinese Academy of Sciences, in 2013. He is currently a research assistant in Institute of Geodesy and Geophysics, CAS, Wuhan, PRC. His research interests include timefrequency analysis, the characteristics of the satellite clock, sparse decomposition, neural network, and Kalman filter. 

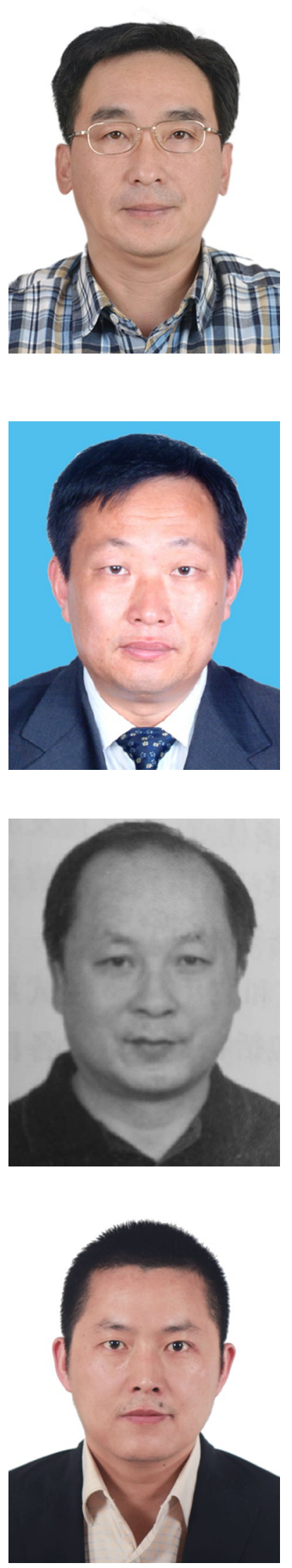

Lintao Liu received the Ph.D. degree of solid geophysics from Chinese Academy of Sciences, in 1999. He went to Geodetic Institute, University Stuttgart, Germany for cooperative research on the support of HumboldtForschungsstipendium from 2001 to 2003 . He is currently a Professor in Institute of Geodesy and Geophysics, CAS, Wuhan, PRC. His research interests are in areas of time-frequency theory and its applications in geodynamics and aviation and marine gravity measurements.

Aigong $\mathrm{Xu}$ is the professor of Liaoning Technical University, Fuxin, China. He received his Ph.D. degree at Wuhan University in 1998. His research focuses on high-precision positioning using GNSS. Due to his great achievements in GNSS applications, he was elected Communication Academician of Russian Academy of natural sciences in 2016.

Feng Pan is currently a senior engineer of Beijing Satellite Navigation Center, Beijing, China. His research interests are in areas of time and frequency characteristic of satellite clock, precise time synchronization and the establishment and keeping of time Reference.

Zhiwu Cai obtained his Ph.D. degree from Information Engineering University, China. He is currently a senior engineer of Beijing Satellite Navigation Center, Beijing, China. His research interests are in areas of the development and application of satellite navigation technology and the establishment and keeping of Time Reference.

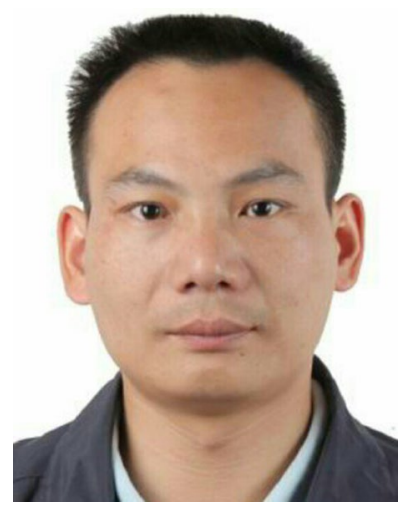

Shenghong Xiao obtained his $\mathrm{Ph}$.D. degree from the Naval University of Engineering, China. He is a senior engineer of Beijing Satellite Navigation Center, Beijing, China. His research interests are in areas of the characteristic of satellite clock, precise time synchronization and the establishment and keeping of time reference.

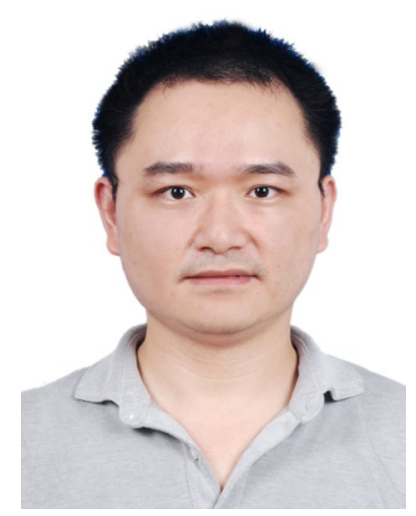

Yi Tu obtained his Ph.D. from University of Chinese Academy of Sciences in 2016. He is currently a lecturer at China Three Gorges University, Yichang, China. His research interests include the application of the GNSS in geodesy, satellite gravimetry and global climate.

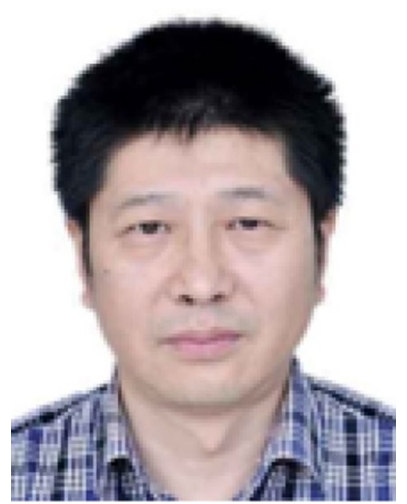

Zhonghua Li is currently a senior engineer of Chinese Academy of Sciences. His research interests mainly focus on time and frequency characteristic of satellite clock and their application in engineering, such as for satellite communication stations and monitoring stations for Beidou system. 